Aus der Abteilung für Liohtbehandlung in Krankenhause

St. Göran, Stookholm (Oberarzt Dr. Magnus Möller).

\title{
Zwei Fälle von Lupus follicularis disseminatus (Tilbury Fox).
}

Von

H. J. Sehlasberg, Stockholm.

(Hiezn Taf. III.)

Im Jahre 1878 beschrieb Tilbury Fox (1) in The Lancet auf der Basis von 3 Fällen ein Krankheitsbild, dem er den Namen: Lupus follicularis disseminatus beilegte. Es handelte sich um einen "Ausschlag" im Gesicht, speziell an der Stirn, den Wangen, dem Kinn und der Gegend um die Augen und dieser bestand in zerstreuten stecknadelkopfgroßen bis halberbsengroßen lupusähnlichen Knötchen von braunroter Farbe und gelatinösem Aussehen, die bei genauer Untersuchung an den Follikeln lokalisiert befunden wurden. Ein Teil der Effloreszenzen, besonders die älteren, waren im Zentrum mit einem gelblichen eiterähnlichen Punkt versehen, der sich doch bei näherer Untersuchung als aus Epithel bestehend herausstellte. Die Knötchen waren indolent, weich anzufühlen und zeigten wenig Anzeichen zu regressiven Veränderungen. Bei oberflächlichem Anblick erinnerten sie an gewöhnliche Akneeffloreszenzen, entbehrten aber ganz des inflammatorischen Aussehens derselben und wurden auch nicht im geringsten durch die Aknetherapie beeinflußt. Die mikroskopische Untersuchung zeigte circumscripte Zellinfiltrate im Corium, zum großen Teil um die oder in der Nähe der Talgdrüsen liegend. Die einzigen Mittel, die sich gegen das Übel wirksam zeigten, waren Caustica. 
Fox meinte, daß eine gleichzertige Disposition für Tuberkulose und Akne für die Entstehung dieser Krankheit erforderlich sei.

Zehn Jahre später (1888) beschreibt Hutchinson (2) dasselbe Übel und bringt für dasselbe die Bezeichnung: A knel up us in Vorschlag. Er meint, es bestehe in einer Kombination von Akne und Lupus entstanden in der Weise, daß die Akneeffloreszenzen von Lupus angegriffen werden. Nur Akneeffloreszenzen im Gesicht sind es, die angegriffen werden, während solche am Rücken frei ausgehen. Hutchins on meint, diese Tatsache illustriere den Sachverhalt, daß Körperteile, die dem Einfluß von Luft und Kälte ausgesetzt sind, für Lupus besonders empfänglich sind.

Von der Poliklinik Unuas beschreibt v. Düring (3) im selben Jahre einen hierhergehörigen Fall unter der Bezeichnung von: Lupus miliaris. Nachdem sich gezeigt hatte, daß die Aknetherapie keinen Nutzen gewährte, wandte Verf. mit gutem Erfolg Salizylsäurekreosotpflaster an. Nach 3 bis 4maliger Applikation des Pflasters fielen die Knötchen heraus unter Hinterlassung ziemlich tiefer roter Narben. Ein bald nachher entstandenes Rezidiv heilte bei Anwendung des Pflasters mit nachfolgender Galvanokaustik. Eine mikroskopiscne Untersuchung wurde nicht vorgenommen.

Ob der Fall, den Besnier (4) in der „Réunion clinique Hebdomadaire des médecins de l'Hospital Saint Louis" in der Zusammenkunft am 6. Dezember 1888 demonstrierte, als Lupus follicularis disseminatus anzusehen ist, ist wohl zweifelhaft. Der Fall, der unter der Bezeichnung von "Lupus tuberculeux, aigu, nodulaire, disséminé" demonstriert wurde, betraf ein 4jähriges Mädchen, Tochter von tuberkulösen Eltern und trat nach überstandenen Masern, in Form von braungelben stecknadelkopfgroßen Knötchen aut, die über den ganzen Körper zerstreut waren, am reichlichsten doch im Gesicht. Eine antiluetische Behandlung hatte nicht deǹ geringsten Einfluß. Die mikroskopische Untersuchung zeigte Zellinfiltrat im Corium, keine Riesenzellen oder Tuberkelbazillen (nur einige zehn Schnitte wurden auf Bazillen untersucht). Ein Meerschweinchen wurde subkutan mit einem excidierten Knötchen eingeimpft und bot nach einem Monat einen palpablen Tuberkel an der Inokulationsstelle dar. Die 11 Wochen nach der Einimpfung bewerkstelligte Sektion des Tieres zeigte allgemeine Tuberkulose.

Dieser Fall unterscheidet sich von anderen beschriebenen teils dadurch, daß es sich hier um ein Kind handelte, teils durch die universelle Ausbreitung der Krankheit. In allen anderen veröffentlichten Fällen ist der Patient über das Pubertätsalter gewesen und ist das Übel auf das Gesicht beschränkt gewesen. Durch das Nichtvorhandensein von Riesenzellen in den Schnitten unterscheidet sich auch der Fall von den übrigen, die Gegenstand mikroskopischer Untersuchung gewesen sind. 
Daß eine Form von Tuberkulose vorgelegen hat, dafür spricht doch deutlich das positive Resultat der Einimpfung.

Sichere Fälle von Lupus follicularis disseminatus sind in der "New-York Dermatological Society“ von Elliot (5) 1889 und G. H. Fox (6) 1890 demonstriert worden. Elliots Fall betraf eine 30jährige Frau, deren Vater, Onkel und Bruder an Tuberkulose gestorben waren. Das Übel hatte zwei Monate zuvor angefangen und bot nun zahlreiche Effloreszenzen dar, besonders an den Wangen und am Kinn. Spontane Involution einiger Effloreszenzen hatte stattgefunden mit Hinterlassung oberflächlicher Narben. Die histologische Untersuchung zeigte "charakteristisch tuberkulöses Gewebe". In der Cutis, gleich unter den Papillen fanden sich kleine Tuberkeln gebildet aus Granulationsgewebe und Riesenzellen, epitheloide Zellen etc. enthaltend. Ein Dutzend Schnitte wurden auf Tuberkelbazillen untersucht, aber mit negativem Resultat. Die Talgdrüsen waren nicht affiziert.

Fox' Patient war ein 32 Jahre alter Mann. Das Übel hatte vor 10 Jahren ganz plötzlich angefangen and sich während dieser ganzen Zeit ziemlich unverändert gehalten. Fox bemerkt, daß die Effloreszenzen mit Leichtigkeit mittels scharfen Löffels exkochleiert werden können, welcher Eingriff doch starke Blutung zur Folge hat.

Ferner teilt Jadasson (7) einen Fall von grofem Interesse mit. Ein an tuberkulöser Periproctitis leidender Mann bietet an Stirn, Wangen, Nase und Kinn einen "Ausschlag" dar, bestehend aus zahlreichen dunkel lividroten bis linsengroßen Knötchen. Klinisch konnte man kaum die Diagnose Lupus stellen, nur die Weichbeit des Gewebes sprach hierfür. Tuberkulinreaktion positiv. Die histologische Untersuchung zeigt. das Bild von Granulationsgewebe mit Langhans Riesenzellen. Die Finimpfung auf Meerschweinchen rief typische Tuberkulose mit protrabiertem Verlauf hervor.

Von der Wiener Schule war Finger (8) der erste, der dieses Übel erwähnt hat. $\mathrm{Ob}$ die von Kaposi (9) unter der Bezeichnung von Acne teleangieatodes beschriebenen Fälle hierher zu rechnen sind, ist wohl zweifelhaft. Finger ist doch geneigt, sie für Lupus follicularis anzusehen.

Finger führt zwei Fälle an; der erste, eine 30jährige Frau, wurde nicht histologisch untersucht, ebensowenig wurden Tierversuche gemacht. Der zweite Fall betraf ein 17jähriges Mädchen, deren Übel vor 3 Monaten anfing. Die Patientin zeigt an den gewöhnlichen Stellen reichlich charakteristische Effloreszenzen. An den Nasenflügeln sind mehrere Knötchen zu größeren braunroten, unebenen, weichen und abschuppenden Infiltraten konfluiert. Eine Tuberkulininjektion von $3 \mathrm{mg}$ gab sowohl allgemeine als lokale Reaktion. Die Aknetherapie war ohne Wirkung. Die Behandlung mit Löffel gab ein schlechtes Resuitat. Freilich konnte man die Infiltrate entfernen und die Substanzverluste heilten rasch mit Hinterlassung von fast unmerklichen Narben, aber um zahlreiche dieser Narben herum bildeten sich neve Knötchen, nach deren Entfernung eine 
neue Eruption entstand. Durch nachfolgende Kauterisation mit "Paquelin" gelang es besser. Man erzielte doch keine vollständige Heilung, sondern dann und wann entstanden neue Knötchen in der Nähe der alten. Einzelne Knötchen, die nicht behandelt worden waren, verschwanden spontan. Die histologische Untersuchung ergab folgendes: Die Epidermis verdünnt, der obere Teil des Stratum papillare nahezu unverändert, um den Haarbalg und die Talgdrüse, teilweise auf ersterem "reitend", findet sich ein scharf begrenztes, von einem schmalen Saum mononucleärer Rundzellen umgebenes Infiltrat, bestehend aws runden "Nestern," die durch mononucleäre und spärliche polynucleäre Runảzellen getrennt sind. Jedes solche "Nest" besteht aus dicht aneinander liegenden epitheloiden Zellen, die eine oder mehrere zentral oder exzentrisch liegende Langhans Riesenzellen einschließen. In den größeren Knötchen findet man bisweilen im Zentrum der Infiltrate einen Herd bestehend aus amorpher, fein granulierter Masse spärliche geschrumpfte Kernreste umschließend ("Verkäsung"). Finge $r$ untersuchte 50 Schnitte von $z$ wei verschiedenen Knötchen auf Bakterien und fand dabei in zwei Präparaten je einen Bazillus, den er auf Grund von Tinktion und Form sich für berechtigt hielt für einen Tuberkelbazillus anzusehen. Zwei Kaninchen wurden in der vorderen Augenkammer und zwei Meerschweinchen, eines subkutan und das andere intraperitoneal mit excidierten Knötchen geimpft, aber mit negativem Resultat.

Balzer und Michaux (10) demonstrierten 1898 in der französischen dermatologischen Gesellschaft einen hierhergehörigen Fall, einen 26jährigen, im übrigen gesunden Mann, ohne tuberkulöse Belastung. Das Übel hatte sich im Laufe von 6 Monaten entwickelt. Eine mikroskopische Untersuchung wurde nicht vorgenommen.

In seinem Werke „Zur Klinik und Histologie seltener Formen der Hauttuberkulose" beschreibt NobI (11) einen Fall von Lupus follicularis disseminatus bei einer 35jährigen Frau. Das Übel hatte vor $1 \frac{1}{2}$ Jahren angefangen und war gelegentlich der Untersuchung nur nach der Stirn lokalisiert. Außer dem Exanthem hatte Pat. eine starke Seborrhé im Capillitium sowie reichlich Komedonen um die Nase. Sie hatte außerdem einen chronischen Lungenspitzenkatarrh und war beleutend anämisch. Die mikroskopische Untersuchung zeigte in der subpapillären Cutisschicht "knotenförmige" Zellanhäufungen getrennt durch schmale kollagene Leisten und umgeben von erweiterten Blutkapillaren. Die Zellen waren teils Rundzellen, teils epitheloide Zellen. Inmitten der Infiltrate fanden sich spärliche Riesenzellen; nicht deutliche käsige Verwandlung. Zahlreiche Schnitte wurden mit negativem Resultat auf Tuberkelbazillen untersucht. Das Übel wurde mit scharfem Löffel und "Paquelin" behandelt und die Substanzverluste heilten in kurzer Zeit. Drei Monate daranf zeigte sich ein Rezidiv mit Knötchen, die an der Grenze der geheilten auftraten. Die Behandlung wurde wiederholt und führte „mutmaßlich definitive Heilung " herbei. Nobl meint, daß die Krankheit durch Tuberkelbazillen mit geschwächter Virulenz hervorgerufen wird. 
Saalfeld (12) teilt einen Fall bei einem 24jährigen Manne mit, völlig ausgebildet nach Verlauf von 2 Monaten. Zwischen den Lupuseffloreszenzen fanden sich gewöhnliche typische Akneeffloreszenzen. Die mikroskopische Untersuchung zeigte teilweis erweiterte Follikeln und Talgdrüsen umgeben von Rundzelleninfiltration. Die Schnitte zeigten außerdem zahlreiche Riesenzellen und käsige Degeneration, Tuberkelbazillen konnten nicht nachgewiesen werden. Einimpfungen auf Kaninchen und Meerschweinchen gaben negatives Resultat. Der Patient wurde mit Cantharidin behandelt, das Übel wurde besser und, sagt Sa alfeld, "es ist zu hoffen, daß bei weiterer Fortsetzung der Kar eine völlige Heilung eintreten wird“. Er ist im übrigen der Meinung, daß Lupus follicularis disseminatus möglicherweise ein Zwischenglied zwischen Lupus vulgaris und Lupus erythematodes bilden kann.

Ein interessanter Fall wurde in der Berliner dermatologischen Gesellschaft am 5. Februar 1901 von Mayer (13) demonstriert. Der Fall ist von Interesse besonders dadurch, daß auch die Nasenschleimhaut angegriffen war. In den übrigen publizierten Fällen sind Veränderungen an den Schleimhäuten nicht erwähnt worden. Hutchinson sagt freilich, daB sein Patient auch Lupus im Gaumen hatte, aber von welcher Form von Lupus die Rede war, wird nicht erwähnt. M a yers Fall betraf ein 20 Jahre altes, kräftig gebautes Mädchen. Eltern und Geschwister gesund. In der Kindheit hatte sie Skrofulose und leidet nun an adenoiden Vegetationen im Nasopharyngealraum. Ihr jetziges Übel datiert sich von vor $1^{1 / 2}$ Jahren. Dasselbe fing in den Nasolabialfalten mit stecknadelkopfgroßen, glänzenden Knötchen von dunkelroter Farbe und weicher Beschaffenheit an. Biopsie wurde nicht gemacht und die Diagnose blieb unklar. Nach $1 / 2$ Jahr kam Pat. verschlimmert zuräck. Sie bot nun außerdem eine größere scheibenförmige Effloreszenz dar, entstanden durch Konfluenz mehrerer kleinerer Knötchen. An der Schleimhaut des Septum narium auf der rechten Seite hat Pat. gleichfalls einige kleine Knötchen, welche von derselben Beschaffenheit wie die an der Haut zu sein scheinen. Eine Effloreszenz an der Haut wurde excidiert und die mikroskopische Untersuchung zeigte klar "das Strukturbild eines tuberkulösen Prozesses". Tuberkelbazillen waren nicht nachzuweisen. May er erwähnt auch einen anderen Fall, den er Gelegenheit, hatte zu beobachten und wo auch die Nasenschleimhaut angegriffen war.

Kreibich (14) demonstrierte einen Fall in der dermatologischen Gesellschaft in Wien bei der Zusammenkunft am 20. Nov. 1901. Es

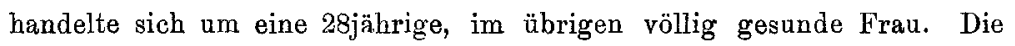
histologische Untersuchung zeigte typisches tuberkulöses Granulationsgewebe mit zentraler Nekrose, epitheloiden Zellen und Riesenzellen. Etwa 50 Schnitte wurden mit negativem Resultat auf Tuberkelbazillen untersucht.

Die jüngste Mitteilung über Lupus follicularis disseminatus haben wir von Bettmann (15), der aus der mediziniseben Klinik in Heidelberg einen Fall beschrieben hat. Das Übel hatte im Juni 1901 angefangen 
und 2 Monate darauf hatte es sich über das ganze Gesicht ausgebreitet. Pat. bot außerdem einige Knötchen an den Fingern und den Unterarmen dar, aber diese waren nach Meinung des Verf, nicht derselben Art wie die im Gesicht. Die mikroskopische Untersuchung eines Knötchens vom Gesicht zeigte folgendes: In der Cutis findet sich ein durch lymphoide Zellen gegen die Umgebung begrenztes rundes Infiltrat, dessen Bau dem des Epitheloidtuberkels entspricht. An manchen Stellen finden sich Langhans Riesenzellen mit regelmäßig angeordneten Kernen. Das Zentrum der Knötchen zeigt käsige Verwandlung. Die Präparate zeigten keineswegs, daB die Entwicklung der Knötehen an die Ungebung der Follikeln gebunden sei. Sechzig Schnitte wurden mit negativem Resuitat auf Tuberkelbazillen untersucht. Verf. machte 3 diagnostische Injektionen mit Alt-Tuberkulin. Eine Probe mit $3 \mathrm{mg}$ wurde negativ, eine Probe mit $5 \mathrm{mg}$ gab ein ungewisses Resultat, dagegen gab eine Probe mit $8 \mathrm{mg}$ sichere lokale und allgemeine Reaktion. Die Einimpfung eines Knötchens in die vordere Augenkammer eines Kaninchens fiel negativ aus. Da einfaches Auskratzen der Knötchen stets Rezidiv im Gefolge hat, bringt Verf. als Behandlung gründliche Exkochleation mit nachfolgender Thermokauterisation in Vorschlag, "aber abgesehen von dem Nachteil der Narbenbildung kann auch diese Behandlung nicht überall Rezidive verhindern".

Ich selbst habe Gelegenheit gehabt, zwei hierhergehörige Fälle zu beobachten, welche in Aubetracht des weniger gewöhnlichen Vorkommens des Übels wohl wert sind veröffentlicht zu werden. Besonders bietet der eine der Fälle mancherlei von Interesse.

Fall I. August Bl., 36 Jahre, ehem. Steinsprenger. Aufgenommen in das Krankenhaus St. Göran den 23./IV. 1904.

Der Vater lebt und ist gesund. Die Mutter ist verstorben, die Todesursache mutmaßilich eine Brustkrankheit; sie war viele Jahre hindurch von Husten und Atemnot belästigt. Ein Bruder des Patienten leidet an Brustkrankheit, zwei andere Geschwister sind gesund. Als Kind hat Pat. die Masern gehabt, im übrigen hat er sich stets einer guten Gesundheit erfreut. Ist niemals von "Finnen" belästigt worden weder im Gesicht noch auf dem Rúcken. Den 13. Januar 1898 wurde Pat. durch einen Sprengschuß am Kopf verletzt, „der ganze Kopf war eine einzige Wunde", beide Augen wurden getroffen und Pat. ist seitdem blind gewesen. Nach 10wöchentlichem Krankenhausaufenthalt wurde Pat. geheilt entlassen. Mit Ausnahme einer größeren ziemlich tiefen Narbe an der linken FrontaIregion und einiger kleineren solchen über dem linken Auge und an der linken Wange bot die Haut im Gesicht damals nichts Bemerkenswertes. Das jetzige Übel des Pat. datiert sich von vor etwas mehr als 1 Jahre. Es fing an als kleine rote Knötchen an der linken Seite der Oberlippe, anf welche bald andere solche folgten lobalisiert nach der Gegend um die Augen. Ungefähr gleichzeitig trat an der Innenseite der Unterlippe 
ein "Ausschlag“ auf, gleichfalls aus kleinen Knötchen bestehend. Das Übel breitete sich rasch aus und nach oinigen Monaten hatte es die Ansbreitung erreicht, die es jetzt zeigt. Subjektive Beschwerden hat Pat. von seinem Übel nicht gehabt. Er ist mit Salben behandelt worden wenngleich ohne Erfolg. Lues wird verneint.

St. pr.: Pat. ist kräftig gebaut, Körperfülle und Muskulatur gut. Die inneren Organe ohne Besonderheiten. Der Harn enthält kein Eiweiß oder Zucker.

An der Stirn, um die Augen, an beiden Wangen, der Oberlippe sowie am Kinn findet sich ein Exanthem bestehend aus stecknadelkopfgroßen bis roggenkorngroßen rundlichen, wenig erhöhten braunroten Knötchen ohne bestimmte Anordnung. Einige sind in der Mitte mit einer kleinen graugelblichen Kruste bedeckt, andere wiederum sind glatt und zeigen in ihrer Mitte eine kleine Vertiefung einer Follikelöffnung entsprechend. Einzelne Knötchen sind durch ein Haar perforiert. Sie sind weich anzufühlen, gegen Druck nicht empfindlich. Bei diaskopischer Untersuchung bleibt ein braungelbes durchsichtiges Infiltrat von gelatinösem Aussehen bestehen. Hier und dort, besonders an den Wangen sind die Knötchen zu größeren und kleineren bis 2pfenniggroßen braunroten, etwas erhöhten, leicht abschuppenden weichen [nfiltraten konfluiert. $Z$ wischen den verschiedenen Effloreszenzen ist die Haut gerötet und etwas geschwollen.

Diese nun beschriebenen Knötchen sind wie angedeutet besonders weich und lassen sich mit Leichtigkeit mittels scharfen Löffels auskratzen, wobei ein mehr oder weniger tiefer scharfrandiger, reichlich blutender Substanzverlust entsteht. Regressive Veränderungen, Ulzeration oder Eiterbildung sind nirgends wahrzunehmen. Die gelbe Kruste an manchen Knötchen simuliert Eiterbildung, wird aber bei mikroskopischer Ontersuchung als nur aus Epithel bestehend befunden. Es sind keine Narben nach spontan involvierten Effloreszenzen nachzuweisen.

An der Schleimhaut der Unterlippe befinden sich 10 bis 12 roggenkorngroße und etwas kleinere unbedeutend erhöhte, schwach durchsichtige Knötchen, der Konsistenz nach sind sie fest, sind scharf begrenzt und gegen die Unterlage frei beweglich. Gegen Druck sind sie etwas empfindlich. Das Epithel über den Knötchen grauweiß, verdickt. Die Schleimhant im Munde im übrigen wie gleichfalls im Rachen und in der Nase ohne Besonderheit. An der Innenseite des linken Daumens hat Pat. einige halberbsengroße blaurote Knötchen, welche doch weder dem Aussehen noch der Beschaffenheit nach denen im Gesicht ähnlich sind. Wann diese Knötchen entstanden sind, kann Pat. nicht angeben, sie sind jedoch mit Sicherheit mindestens $1 \mathrm{Jahr}$ bevor der "Ansschlag" im Gesicht auftrat, vorhanden gewesen.

Pat. war aus der Provinz hereingesandt worden als verdächtig an Lupus zu leiden, um der Lichtbehandlung nach $F$ i n s en unterzogen zu werden. Die Diagnose war anfänglich nicht 
leicht zu stellen. Daß von der gewöhnlichen Form von Lupus nicht die Rede sein konnte, war beim ersten Anblick des Patienten klar. Eine Verwechslung mit Acne vulgaris war auch nicht möglich. Dagegen erinnerte das Übel recht sehr an ein papulöses Syphilid. Die Weichheit des Gewebes sowie die diaskopische Untersuchung sprachen doch dafür, daß die Knötchen von derselben Beschaffenheit waren wie diejenigen, die man bei Lupus vulgaris sieht.

$\mathrm{Zu}$ diagnostischen $\mathrm{Zwecken}$ erhielt Pat. den 25. IV. eine Injektion von $1 \mathrm{mg}$ Alt-Tuberkulin. Es zeigte sich keine weder lokale noch allgemeine Reaktion. Den 29./IV. erhielt Pat. eine Tuberkulininjektion von $5 \mathrm{mg}$ mit gleichem Resultat. $\mathrm{Er}$ erhielt nun 14 Tage hindurch täglich $6 g$ Jodkalium, während außerdem eine talergroße Sammlung von Effloreszenzen mit Hg-Pflaster bedeckt wurde. Bei Beendigung dieser Behandlung war keine Veränderung wahrzunehmen mit Ausnahme des mit dem Pflaster behandelten Gebietes, das doch nur Mazeration der Epidermis darbot.

Um durch mikroskopische Untersuchung über die Diagnose Klarheit zu erhalten wurde den 2./V. ein Knötchen von der Haut und eines von der Schleimhaut der Unterlippe excidiert. Die Präparate wurden in Alkohol gehärtet und in Paraffin eingebettet. Die Excisionswunden heilten per primam.

Den 17./V. bekam Pat. Erysipelas faciei, das sich in 2 Tagen über das ganze Gesicht und Kapillitium ausbreitete. Die Temperatur war während der nächsten Tage bis $40^{\circ}$ hinauf und der Gesamtzustand war ziemlich schlecht. Es gelang doch das Übel zu begrenzen und nach etwa einer Woche war Pat. wieder munter. Man bemerkte nun, daß die Gesichtshaut etwas bleicher geworden war und, daß manche der Knötchen gleichsam zusammengesunken waren und sich wenig über die umgebende Haut erhoben. Pat. wurde nun einige Zeit nur mit Resorzinumschlägen behandelt, welche Behandlung, wie natürlich zu erwarten stand, nicht den geringsten Effekt hatte.

Den 6./VI. erhielt Pat. $10 \mathrm{mg}$ Tuberkulin. Er reagierte nun mit einer Temperatur zwischen 38 bis $39^{\circ}$ sowie Kopfweh und allgemeinem Unwohlsein. Eine sichere lokale Reaktion konnte doch nicht wahrgenommen werden. Möglicherweise zeigten sich die Effloreszenzen etwas mehr succulent, aber im übrigen verhielten sie sich wie zuvor.

Die paraffingebetteten Schnitte wurden teilweise in Serien geschnitten and mit Hämatoxylin-Eosin, polychromem Methylenblau und um das Elastin darzustellen nach Unna-T aenzer gefärbt.

Die histologische Untersuchung gab folgendes Resultat:

a) Die Effloreszenz von der Haut: Bei mäßiger Vergrößerung sieht man im Corium zerstreute, scharf begrenzte, 
vorwiegend runde Zellinfiltrate, von denen manche gleich unter dem Stratum papillare, unmittelbar an den oder in der Nähe der Follikeln gelegen sind, andere wiederum liegen ticfer, entweder in der Nähe von Schweißdrüsen oder auch frei im Bindegewebe. Zahlreiche Riesenzellen sind in den meisten der Infiltrate zu sehen.

So ist das Bild in Schnitten von der Randpartie des Präparates. In dem Maße, als die Schnitte sich dem Zentrum nähern, werden die Infiltrate größer und schmelzen allmählich zusammen.

Bei stärkerer Vergrößerung zeigt sich, daß die Infiltrate aus einer retikulär angeordneten Stützsubstanz bestehen, in der sich hauptsächlich epitheloide Zellen, spärliche lymphoide Rundzellen und Riesenzellen von $L$ ang han s Typus eingebettet finden. Die Peripherie der Infiltrate besteht aus Rundzellen, während sich die Riesenzellen im allgemeinen im Zentrum finden. Außer den nun erwähnten Zellformen findet man in den mit polychromem Methylenblau gefärbten Schnitten ziemlich zahlreiche Plasmazellen und einzelne "Mastzellen". Es sind keine Gefäße, auch kein elastisches Gewebe in den Infiltraten wahrzunehmen. In den größeren befinden sich bisweilen im Zentrum kleinere Flecke, die die Farbe schlecht angenommen haben und die aus einer amorphen Masse nebst Kernresten (käsige Verwandlung) zu bestehen scheinen. Außerhalb der Infiltrate zeigt das Corium keine gröberen Veränderungen. Die Gefäße sind erweitert und hin und wieder sieht man sie von Kleinzelleninfiltration umgeben. Die Follikel scheinen auch größer als normal zu sein. Wo der Prozeß seinen Höhepunkt erreicht, d. h. im Zentrum des Knötchens sind die Papillen völlig gestrichen, ebenso die Retezapfen, und das Infiltrat tritt nahezu völlig an das verdünnte Epithel heran, dieses ist auch überall beibehalten. Das elastische Gewebe ist um die Infiltrate herum vermehrt ersichtlich darauf beruhend, daß es beim Heranwachsen des Infiltrates verdrängt worden ist. Einen direkten Zusammenhang zwischen Gefäßen und Infiltraten nachzuweisen, ist mir trotz genauer Durchsicht von Serien von Präparaten nicht gelungen.

Das mikroskopische Bild stimmt demnach der Hauptsache nach mit dem überein, was zuvor von anderen Fällen von Lupus follicularis disseminatus beschrieben worden ist. Wir haben bier das Bild eines typischen epitheloiden Tuberkels mit nachfolgenden sekundären Veränderungen.

b) Das Knötchen von der Schleimhaut der Unterlippe:

Das mikroskopische Bild gleicht dem unter a) beschriebenen natürlich mit den Modifikationen, die die Lokalisation in einer Schleimhaut bedingt. Die Infiltrate sind doch reich- 
licher und füllen die Mucosa nahezu ganz. Eine nähere Beschreibung wäre nur eine Wiederholung von dem, was zuvor gesagt worden ist, weshalb solche nicht vonnöten ist.

Einige 50 Schnitte von jedem der Knötchen wurden nach $\mathrm{Zi}$ e h l-Ne elsen (Karbolfuchsin-Salpetersäure-AlkoholMethylenblau) auf Tuberkelbazillen untersucht, aber mit negativem Resultat.

Da das gebärtete Material wicht ausreichte und in dem Gedanken, daß die Härtung und Einbettung möglicherweise den Nachweis der Bazillen erschweren könne, bediente ich mich für weitere Untersuchungen des ausgekratzten Inhalts eines Teiles der Effloreszenzen (dies gilt nur von solchen an der Haut), der sich mit Leichtigkeit zerstückeln und zwischen zwei Objektgläsern ausbreiten ließ. Um Verunreinigungen zu vermeiden, wurde die größtmögliche Vorsicht beobachtet. In solcher Weise wurde der Inhalt in 7 Effloreszenzen, auf 14 Präparate verteilt, untersucht. In 12 von den Präparaten fanden sich keine Tuberkelbazillen, auch keine andere Bakterien, in einem Präpatat fand ich eine Gruppe von 7 Bazillen, die auf Grund des Aussehens und des Färbungsverhaltens für Tuberkelbazillen angesehen werden $\mathrm{mu}$ uten. Vier von diesen Bazillen lagen in einer großen Zelle, möglicherweise einer Riesenzelle, eingeschlossen, die übrigen lagen in der Umgebung derselben zerstreut. In einem andern Präparat fand ich einen einzigen Bazillus, den vorhergehenden völlig gleich.

Um die Diagnose des weiteren zu prüfen, wurden Tierexperimente vorgenommen. Ein Kaninchen wurde in der vorderen Augenkammer mit einem excidierten Knötchen geimpft. Außerdem wurde ein Meerschweinchen intraperitoneal mit dem Inhalt in 3 Effloreszenzen geimpft, der gut zerteilt mit einem $\mathrm{cm}^{3}$ sterilem Wasser ausgeriihrt worden war. Die Sektion der Versuchstiere wurde 11 Wochen nach der Einimpfung vorgenommen. Es war indessen nirgends ein Zeichen von Tuberkulose wahrzunehmen. Wenn demnach bei der Impfung Tuberkelbazillen in den Tierorganismus eingeführt worden waren, sind diese von so geschwächter Virulenz gewesen, daß sie nicht im stande gewesen sind, tuberkulöse Veränderungen in demselben hervorzurufen.

Nachdem die Diagnose durch die mikroskopische Untersuchung klar geworden war, durfte der Pat. mit Lichtbehandlung (nach Fin sen) anfangen. Auf Grund der von einem zuvor behandelten Fall gewonnenen Erfahrung werden die Effloreszenzen erst gekratzt und gebrannt (Galvanokaustik), bevor 
man das Licht einwirken läßt. Eine kräftige Reaktion tritt ein und die Heilung geht sicher, wenn auch langsam, vorwärts. Die Knötchen an der Schleimhaut, die ja nicht Gegenstand der Lichtbehandlung werden können, werden mit Galvanokaustik behandelt. Pat. ist fortgesetzt in Behandlung und es sind gute Aussichten dafür vorhanden, daß er völlig geheilt werden wird.

Fall II. ${ }^{1}$ ) Adolf C., 30 Jahre. Zuckersiedereiarbeiter. Eingetragen in der Abteilung für Lichtbehandlung 11./II. 1903, geheilt entlassen 30./VI. 1903.

In hereditärer Beziehung nichts Bemerkenswertes. Pat. hat sich stets einer guten Gesundheit erfreut. Das gegenwärtige Leiden fing vor 6 Monaten an und erreichte in kurzer Zeit die Ausbreitung, die es jetzt zeigt. Behandelt mit Salben, obgleich ohne Erfolg.

St. pr. Ziemlich kräftige Körperkonstitution. Körperfülle und Muskulatur gut. Lungen ohne nachweisbare Veränderungen. Sichtbare Schleimhäute ohne Besonderheit. Keine subjektiven Beschwerden.

An der Stirn, den Augenlidern, der Nase, den Wangen, den Ohren und dem Kinn hat Pat. ein Exanthem bestehend ans zerstreuten bis roggenkorngroßen Knötchen von braunroter Farbe, die sich etwas über das Hautniveau erheben. Dieselben sind nicht gegen Druck empfindlich, sind weich anzufüblen und sind leicht mit einer Knopfsonde zu durchbohren. Bei diaskopischer Untersuchung bleibt ein scharf begrenztes braungelbes Infiltrat bestehen. Ein Teil der Knötchen sind deutlich follikulär mit kraterförmigem Zentrum; andere sind in der Mitte mit leicht abzulösenden graugelben Schuppen bedeckt. An den Augenlidern sind einige Effloreszenzen zu größeren Infiltraten konfluiert. Nirgendwo irgendwelche Anzeichen von Ulzeration. Einige Effloreszenzen in der Gegend der Augenbrauen zeigen Anzeichen von spontaner Involution, sie sind etwas eingesenkt, dunkler braunpigmentiert und mehr abschuppend.

Die von Möller bewerkstelligte histologische Untersuchung ergibt Infiltrat um die Haarfollikeln herum mit zahlreichen Plasmazellen und einzelnen Riesenzellen. Einige zehn Schnitte wurden mit negativem Resultat auf Tuberkelbazillen untersucht. Tierversuche wurden nicht gemacht. Tuberkulininjektionen von 1 and $2 \mathrm{mg}$ gaben negatives Resultat. Nach einer Injektion von $10 \mathrm{mg}$ ging die Temperatur bis $38^{\circ} 2^{\circ}$ hinauf, im übrigen keine allgemeine, noch auch lokale Reaktion.

Gleich nach Eintreffen wurde mit Lichtbehandlung nach Finsen begonnen. Diese zeigte sich doch wenig wirksam und es ging sehr langsam mit der Heilung. Um sicherer Lues ausschließen zu können, erhielt Pat. gleichzeitig Kalomelinjektionen. Irgendwelche Wirkung der $\mathrm{Hg}-\mathrm{Be}-$ handlung (5 Injektionen à $0 \cdot 10 \mathrm{ctg}$ ) konnte doch nicht wahrgenommen

1) Vergl. Mag nus Möller (19) Mitteilungen aus der Abteilung für Lichtbehandlung im Krankenhause St. Göran, Stockholm. Fall 16. Nord. Med. Arkiv 1904, Abt. II. Heft 3. Nr. 10.

Arch. f. Dermat. u. Syph. Bd. LXXIV. 
werden. Erst nachdem die Behandlung in der Weise geändert worden war, daß die Knötchen, bevor sie dem Lichte ausgesetzt wurden, mit Galvanokaustik behandelt wurden, ging die Heilung rasch vorwärts und nach $4 \frac{1}{2}$ monatlicher Behandlung konnte Pat. als geheilt entlassen werden. Er hatte dann 148 Belichtungen erhalten. Es ist nun (Sept. 1904) über 14 Monate her, seit die Behandlung beendigt wurde, aber ein Rezidiv hat sich noch nicht gezeigt.

Lupus follicularis ist eine ziemlich ungewöhnliche Form von Hauttuherkulose. Im vorstehenden habe ich alle mir zugänglichen zuvor veröffentlichten Fälle besprochen. Die Mehrzahl der Verfasser verfügt nur über je einen Fall. Tilbury F ox beschrieb die Krankheit an der Hand von 3 Fällen.

Sie greift besonders jüngere Leute an und ihr Auftreten ist immer so gut wie akut oder subakut. Im Laufe einiger Wochen oder Monate hat sie ihre volle Entwicklung erreicht. Sie zeigt sich in Form von zerstreuten braunroten oder bisweilen lividroten stecknadelkopfgroßen bis halberbsengroßen Knötchen, die sich etwas über die Umgebung erheben. Manche der Knötchen, besonders die älteren, sind bisweilen mit einem graugelben Punkt in der Mitte versehen, der, wie sich bei mikroskopischer Untersuchung zeigt, nur aus Epithel besteht. Manche Effloreszenzen bieten in der Mitte eine Follikelöffnung dar, andere wiederum sind von einem Haar durchbohrt. Gewöhnlich kommen die Knötchen isoliert vor, bisweilen können sie wie in meinem Falle I zu größeren oder kleineren Plaques konfluieren. Die umgebende Haut ist im allgemeinen unverändert, bleich; in meinem Fall I war sie etwas gerötet und geschwollen. Die diaskopische Untersuchung zeigt ein braungelbes durchsichtiges Infiltrat von gelatinösem Aussehen. Die Knötchen sind nicht gegen Druck empfindlich, weich anzufühlen, und lassen sich mit Leichtigkeit mit scharfem Löffel auskratzen, wobei sie einen mit scharfen Rändern versehenen, stark blutenden Substanzverlust zurücklassen.

Zum Unterschied von Lupus vulgaris bleiben in den meisten Fällen die Schleimhäute unberührt. $\mathrm{Da} B$ diese doch auch angegriffen werden können, zeigen $\mathrm{M}$ a yers zwei Fälle und besonders mein Fall I, wo das mikroskopische Bild deutlich zeigte, daß die Knötchen an der Innenseite der Unterlippe von derselben Beschaffenheit waren wie die auf der Haut. 
Obwohl das klinische Bild dieser Krankheit charakteristisch genug ist, ist man doch nicht berechtigt, ausschließlich auf Grund desselben eine sichere Diagnose zu stellen. Um ein Hautleiden als tuberkulös bezeichnen zu können, sind nach Jadassohn (16) folgende Punkte zu berücksichtigen: 1. der Nachweis von Tuberkelbazillen in den Krankheitsprodulsten; 2. die Möglichkeit mit den Krankheitsprodukten bei empfänglichen Tieren eine Inokulationstuberkulose hervorrufen zu können; 3. die histologische Struktur; 4. das klinische Aussehen und der Verlauf der Krankheit; 5. die Kombination mit anderen sicher tuberkulösen Erscheinungen und das Vorkommen hereditärer Belastung; 6. die Reaktion auf Tuberkulin.

Unter den seither veröffentlichten Fällen von Lupus follicularis findet sich keiner, der in allen diesen Punkten positiven Ausschlag gegeben hätte. Die Verfasser haben im allgemeinen nur auf Grund des klinischen Bildes und mit Hilfe der mikroskopischen Untersuchung ihre Diagnose gestellt. In einigen Fällen sind auch andere Gesichtspunkte in Betracht gezogen worden. Tuberkulininjektionen sind rorgenommen worden ron Jadassohn, Finger, Bettmann und mir. Jadassohns Injektion gab positives Resultat, eine wie große Dosis angewendet wurde, ist doch nicht erwähnt. F in g er erhielt positives Resultat von $3 \mathrm{mg}$. Bettmanns Fall gab bei einer Dosis von $1 \mathrm{mg}$ negatives Resultat, bei Injektion von $5 \mathrm{mg}$ wurde das Resultat ungewiß, erst $8 \mathrm{mg}$ gaben sichere sowohl lokale als allgemeine Reaktion. In meinem Falle I wurden sowohl $1 \mathrm{mg}$ als $5 \mathrm{mg}$ ohne Effekt injiziert, bei Injektion von $10 \mathrm{mg}$ wurde allgemeine Reaktion erzielt, aber keine sichere lokale solche. In Fall II wurde gleichfalls keine Lokalreaktion erzielt.

Die Ursache der negativen Resultate der Tuberkulininjektionen in meinen Fällen kann ich nicht erklären. Dieselbe Tuberkulinlösung wurde auf mehrere Fälle von Lupus vulgaris angewendet und gab hier lokal positives Resultat, so daß irgendwelche Fehlerhaftigkeit der Injektionsflüssigkeit sicher ausgeschlossen werden kann. Nach Jadassohn (16) beweisen doch negative Resultate von Tuberkulininjektionen gar nichts, denn es gibt einige tuberkulöse Hautleiden, welche relativ oft nicht reagieren wie z. B. Tuberculosis verucosa cutis. Eine 
deutliche lokale Reaktion dagegen macht die Diagnose außerordentlich wahrscheinlich. Ähnliche Beobachtungen sind gemacht worden von Grouven (17) u. a. m. Indessen sind fortgesetzte Beobachtungen hierüber von größtem Interesse.

Eine mikroskopische Untersuchung ist in recht vielen Fällen vorgenommen worden. Til bury Fox fand circumscripte Zellinfiltrate im Corium ringsum die oder in der Nähe der Talgdrüsen. Elli ots Fall zeigte kleine Tuberkeln von Granulationsgewebe, die Riesenzellen, epitheloide Zellen etc. enthielten. Die Talgdrüsen waren nicht affiziert. Jadassohn fand Granulationsgewebe mit Riesenzellen, Finger epitheloide Tuberkeln, ebenso Nobl, Sa alfeld fand Infiltrate mit Riesenzellen und käsiger Degeneration, Mayer und Kreibich typisches tuberkulöses Granulationsgewebe und Bettmann epitheloide Tuberkeln. Bettmanns Präparate zeigten deutlich, daß der Prozeß nicht an die Follikeln gebunden ist. Meine Präparate stimmen der Hauptsache nach mit dem überein, was zuvor beobachtet worden ist. Gleichfalls zeigen sie, daß die Infiltrate sich unabhängig von den Follikeln entwickeln können. Eine mikroskopische Untersuchung von der Schleimhaut ist nur in meinem Fall I gemacht worden und die Bilder zeigen auch hier typische Tuberkeln.

Untersuchungen auf Tuberkelbazillen in Schnitten sind vorgenommen worden von Elliot, Nobl, Saalfeld, Mayer, Kreibich und Bettmann, alle mit negativen Resultaten. Finger fand in zwei Präparaten je einen Tuberkelbazillus. Meine eigenen Untersuchungen von Schnitten gaben negatives Resultat. Bei direkter Untersuchung des Ausgekratzten von Hauteffloreszenzen gelang es mir dagegen sichere Tuberkelbazillen nachzuweisen. In Anbetracht des großen Materials, das dabei untersucht wurde, ist es klar, daß Tuberkelbazillen nur in äußerst geringer Menge bei dieser Form von Hauttuberkulose rorkommen.

Wenn man den Fall Besniers ausnimmt, ist ein positives Impfungsresultat an Tieren nur von $\mathrm{Jadassohn}$ erzielt worden. Nähere Details von dem Impfungsversuch werden doch nicht mitgeteilt. 
Tilbury Fox war der Meinung, daß eine gleichzeitige Disposition für Tuberkulose und Acne vulgaris erforderlich sei für die Entstehung von Lupus follicularis disseminatus. Dieselbe Ansicht hegte auch $H u t c h i n s o n$. Nach seiner Meinung entsteht das Leiden dadurch, daß die Akneeffloreszenzen von Lupus angegriffen werden. Aus den Krankengeschichten der anderen Verfasser geht hervor, daß einige der Patienten im übrigen völlig gesund waren, andere zeigten Tuberkulose in anderer Form oder auch tuberkulöse Disposition. Meine eigenen Patienten wurden genau untersucht, speziell in Bezug auf Tuberkulose, ohne daß doch etwas Verdächtiges zu entdecken war. (In Fall I liegt möglicherweise Disposition zu Tuberkulose vor.) Ebeusowenig fanden sich bei ihnen gewöhnliche Akneeffloreszenzen weder jetzt noch laut Angabe vor Auftreten des gegenwärtigen Leidens. Die mikroskopische Untersuchung spricht ja auch dafür, daß die Infiltrate unabhängig von den Follikeln entstehen können. Die Bezeichnung "follicularis" ist vielleicht auch nicht völlig berechtigt.

Wie läßt sich die Entstehung der Krankheit erklären? Wahrscheinlich ist, daß von einem inneren Herd Tuberkelbazillen in das Blut hineingelangen und dann zu Embolien Anlaß geben. Das akute Auftreten spricht dafür, daß der Prozeß ungefähr so zugehen muß. Daß es gerade das Gesicht ist, das der Sitz der Invasion wird, ist ja recht eigentümlich. Vielleicht, daß der Einfluß des Lichtes und der Luft irgend eine Rolle dabei spielt. Einen anatomischen Anhaltspunkt für diese Hypothese durch den Nachweis vermittelnder Gefäßveränderungen habe ich, trotzdem ich die Aufmerksamkeit hierauf gerichtet hatte, nicht geben können. Wo lters (18) hat jüngst einen Fall von Lupus veröffentlicht, wo es ihm gelungen war, den hämatogenen Ursprung der Krankheit nachzuweisen.

Das Leiden, welches am leichtesten zu Verwechslung mit Lupus follicularis Anlaß geben kann, ist das acneiforme Syphilid. Das Resultat der antiluetischen Behandlung sowie die mikroskopische Untersuchung müssen hier den Ausschlag geben. Fine Verwechslung mit Acne vulgaris, die in manchen Fällen stattgefunden hat, muß mit Leichtigkeit zu vermeiden sein. 
Wenn das Übel sich selbst überlassen bleibt, kann es lange Zeit hindurch unverändert stehen bleiben. In Fox Fall hatte der Pat. sein Übel 10 Jahre lang gehabt, ohne daß dasselbe die geringste Veränderung erfahren hatte. Ulzeration, Eiterbildung oder andere klinisch nachweisbare Prozesse kommen nicht vor. Bisweilen trifft es doch ein, daß eine und die andere Effloreszenz spontan involviert wird mit Hinterlassung einer oberflächlichen Narbe (wie in meinem Falle II).

Zum Schluß ein paar Worte über die Behandlung der Krankheit. Schon Tilbury Fox erklärt die Aknetherapie in derartigen Fällen für gänzlich unbrauchbar. Nur von Caustica kann man einigen Erfolg erwarten. v. Düring wandte Salizylkreosotpflaster an. das doch mit Galvanokaustik kombiniert werden mußte, um die beabsichtigte Wirkung zu erzielen. Fing er wandte anfänglich nur Auskratzung der Effloreszenzen an, was zur Folge hatte, dafj er bald Rezidiv erhielt. Seitdem er auf die Auskratzung Kauterisierung mit "Paquelin" folgen lie日, wurde das Resultat besser. Dieselbe Behandlung wurde auch von Nobl angewendet. Saalfeld wandte Behandlung mit Kantharidin an, in welcher Form ist doch nicht erwähnt. Das Resultat der Behandlung ungewiß. Bettmann wandte auch Auskratzung nebst Kauterisation an, aber "die Narbenbildung unberücksichtigt, kann ein Rezidiv dadurch nicht verhindert werden". Eine Behandlung, die gute Resultate zu gewähren scheint, ist die Lichtbehandlung nach Finsen mit vorhergehender Auskratzung und Galvanopunktur der Effloreszenzen. Fall II wurde so völlig geheilt nach 149 Seancen mit hübschen oberflächlichen, wenig bemerkbaren Narben. Ein Rezidiv hat sich, trotzdem mehr als $1 \mathrm{Jahr}$ seit der Beendigung der Behandlung verflossen ist, nicht gezeigt. Ein Versuch, die Hauteffloreszenzer ohne vorhergehende Auskratzung mit Licht $\mathrm{zu}$ behandeln, fiel weniger günstig aus, das Licht schien nur unbedeutenden Einfluß zu haben und die Heilung blieb ungeachtet aller Energie bei der Behandlung aus. Die Behandlung mit Licht ist freilich recht zeitraubend und kostspielig, aber in Anbetracht, daß sie große Garantie für das Ausbleiben von Rezidiven gewährt und daß sie ein hübsches Resultat in Be- 
zug auf die Narbenbildung verspricht, verdient sie wohl angewendet $\mathrm{zu}$ werden.

Herrn Doktor Magnus Möller gestatte ich mir meinen herzlichen Dank auszusprechen für seine Bereitwilligkeit, mir das nötige Material zur Verfügung zu stellen, sowie für das rege Interesse, das er meiner Arbeit gewidmet hat.

\section{Literatur.}

1. Tilbury Fox. Clinical lecture on disseminated follicular lupus (simulating acne). The lancet. 1878.

2. Hutchinson. Harvein lectures on lupus. The British medical journal 1888.

3. v. Dü ring. Ein Fall von "Lupus miliaris" oder sogenanntem „Aknelupus.“ Monatshefte für Prakt. Dermatol. 1888.

4. B es nier. Lupus tuberculeux aigu, nodulaire, disséminé. Annales de dermat. et $d \rightarrow$ syphiligr. 1889.

5. Elliot. Lupus disseminatus. Journal of cutaneous and genitourinary diseases. 1889.

6. G. H. Fox. Colloid milium. Journal of cutaneous and genitourinary diseases. 1890 .

7. Jadassohn. Über Lupus oder Acne teleangiectodes. Vortrag gehalten in der medizinischen Sektion der schlesischen Gesellschaft für vaterländische Kaltur, 26. Januar 1894. Zit. nach Fing er.

8. Finger. Über Lupus follicularis disseminatus (Tilbury Fox), Acne teleangiectodes (Kaposi). Wiener klinische Wochenschrift 1897.

9. Kaposi. Pathologie und Therapie der Hautkrankheiten. Berlin u. Wien. 1899 .

10. Balzer u. Michaux. Lupus de la face à nodules miliaires disséminés. Annales de dermatol. et de syphiligr. 1898.

11. Nobl. Zur Klinik und Histologie seltener Formen der Hauttuberkulose. Festschrift gew. Moritz Ka posi. Ergänzungsband zum Arch. für Derm. u. Syph. 1900.

12. Sa alfeld. Über Lupus follicularis disseminatus und über die Beziehungen zwischen Lupus vulgaris und Lupus erythematodes. Derm. Zeitschr. 1901.

13. Mayer, Demonstration in der "Berliner dermatol. Gesellschaft, 5. Febr. 1§u1. Dermatol. Zeitschr. 1901. 
14. Kreibich. Demonstration in der "Wiener derm. Gesellschaft" 20. Nov. 1901. Arch. f. Derm. u. Syph. 1902.

15. Bettmann. Lupus follicularis disseminatus. Beiträge zur Klinik der Tuberkulose. Heft I. Würzburg 1903.

16. Jadassohn. Die tuberkulösen Erkrankungen der Haut. Ergebnisse der speziellen patholog. Morphologie a. Physiologie der Sinnesorgane. Wiesbaden 1896.

17. Grouven. Ein Fall von tuberkulösem Tumor der Glans penis. Arch. f. Derm. u. Syph. 1904. Bd. LXX. Heft 2.

18. Wolters. Über einen Fall von Lupus nodularis hämatogenen Ursprungs. Arch. f. Derm. u. Syph. 1904. Bd. LXIX. Heft 1. u. 2.

19. M a grus Möller. Mitteilungen aus der Abteilung für Lichtbehandlung im Krankenhause St. Göran. Nord. med. Arkiv. 1904. Abt. II, Heft 3, Nr. 10.

\section{Die Erklärung der Abbildung auf Taf. III ist dem Texte zu entnehmen.}




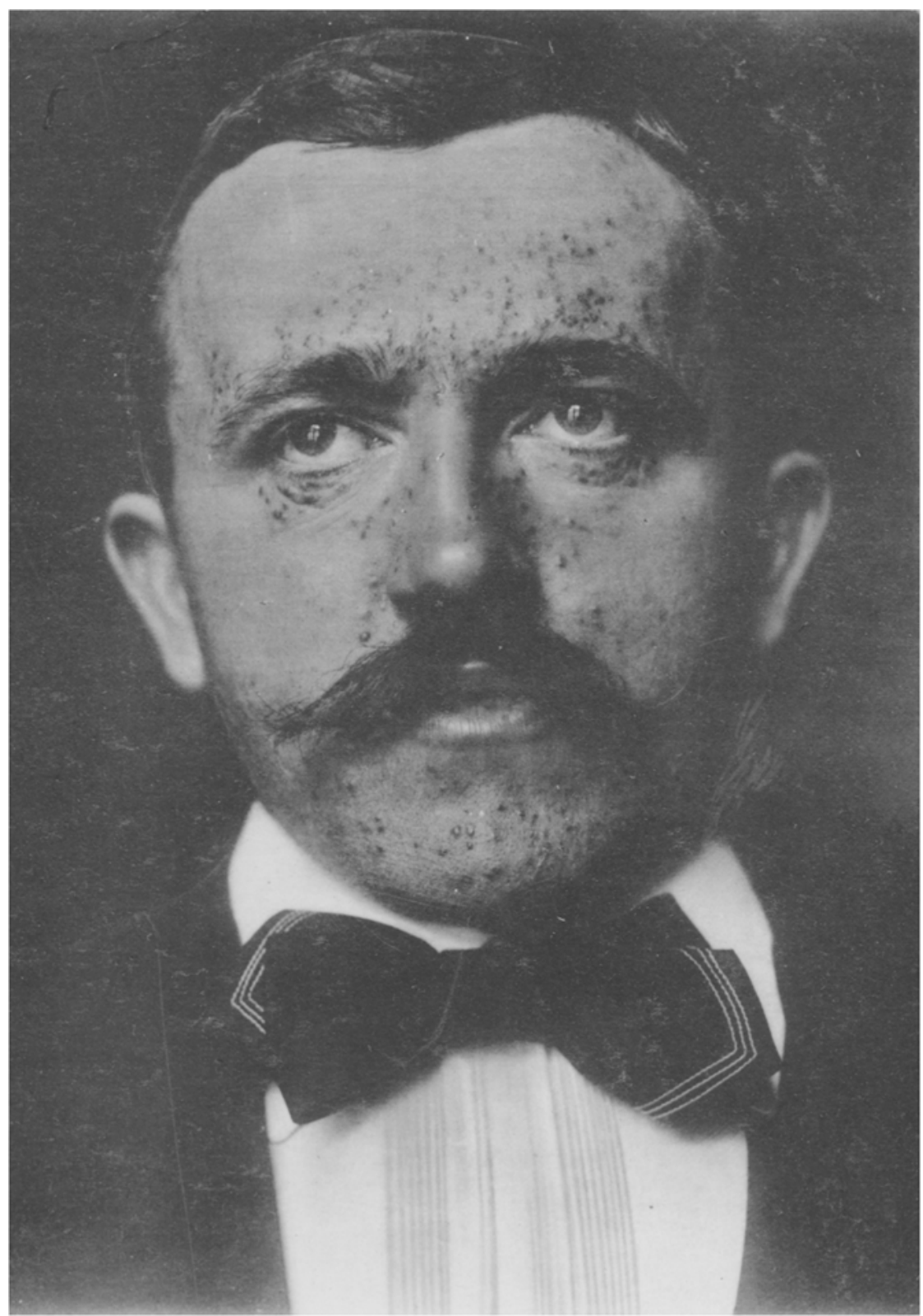

Schlasberg: Lupus follicularis dissem. 